\title{
Dielectric Relaxation of Biological Water ${ }^{\dagger}$
}

\author{
Nilashis Nandi and Biman Bagchi*, \\ Solid State and Structural Chemistry Unit, Indian Institute of Science, Bangalore, India 560012
}

Received: June 9, 1997; In Final Form: September 30, 1997

\begin{abstract}
Dielectric relaxation and NMR spectrum of water in biological systems such as proteins, DNA, and reverse micelles can often be described by two widely different time constants, one of which is in the picosecond while the other is in the nanosecond regime. Although it is widely believed that the bimodal relaxation arises from water at the hydration shell, a quantitative understanding of this important phenomenon is lacking. In this article we present a theory of dielectric relaxation of biological water. The time dependent relaxation of biological water is described in terms of a dynamic equilibrium between the free and bound water molecules. It is assumed that only the free water molecules undergo orientational motion; the bound water contribution enters only through the rotation of the biomolecule, which is also considered. The dielectric relaxation is then determined by the equilibrium constant between the two species and the rate of conversion from bound to free state and vice versa. However, the dielectric relaxation in such complex biomolecular systems depends on several parameters such as the rotational time constant of the protein molecule, the dimension of the hydration shell, the strength of the hydrogen bond, the static dielectric constant of the water bound to the biomolecule, etc. The present theory includes all these aspects in a consistent way. The results are shown to be in very good agreement with all the known results. The present study can be helpful in understanding the solvation of biomolecules such as proteins.
\end{abstract}

\section{Introduction}

Many biological systems such as proteins and enzymes are inactive without water. For a complete knowledge of the function of such systems, an understanding of the structure and dynamics of the aqueous environment surrounding the concerned biomolecule is thus essential. The properties of water molecules in the vicinity of a biomolecule differ appreciably from those of bulk water. ${ }^{1-4}$ The water molecules enclosed within the solvation shell present in the immediate vicinity of the biomolecule are termed "biological water". The dynamics and structure of biological water near proteins, DNA, and in reverse micelles have been the subject of intense research over several decades. ${ }^{1-28}$ Dielectric spectroscopy and nuclear magnetic resonance spectroscopy (NMR) are the two most extensively used techniques to understand the interaction of water with proteins. Both of these two methods essentially probe molecular orientational relaxation. It is now known that the hydration shell surrounding a protein molecule comprises different types of water. ${ }^{1-3}$ Few water molecules remain rigidly bound to the protein for a very long time. In the immediate vicinity of the surface of the protein, there are water molecules that experience much faster rotational and translational diffusion rate than the water molecules directly bound to the biomolecule. Thus, biological water is believed to consist of two kinds of water molecules, usually referred to as "bound" and "free", depending on their momentary states of existence. There is, of course, dynamic exchange between the two species.

On the basis of the experimental studies of the dynamic behavior of water near biomolecules, it has been established that the frequency dependent dielectric constant of the combined biomolecule-water system can be written as a sum of four

* To whom correspondence should be addressed. E-mail: bbagchi@ sscu.iisc.ernet.in. FAX: 91-80-3341683; 91-80-3311310.

$\dagger$ Dedicated to our teacher Professor Mihir Chowdhury on his 60th birthday.

*Also at Jawharlal Nehru Center for Advanced Scientific Research, Bangalore, India.

${ }^{\otimes}$ Abstract published in Advance ACS Abstracts, November 15, 1997. dispersion terms as follows: ${ }^{5 \mathrm{a}, 8 \mathrm{k}}$

$$
\epsilon(\omega)=\epsilon_{\infty}+\frac{\Delta_{1}}{1+\mathrm{i} \omega \tau_{1}}+\frac{\Delta_{2}}{1+\mathrm{i} \omega \tau_{2}}+\frac{\Delta_{3}}{1+\mathrm{i} \omega \tau_{3}}+\frac{\Delta_{4}}{1+\mathrm{i} \omega \tau_{4}}
$$

with $\epsilon_{\infty}$ denoting the infinite frequency dielectric constant of bulk water, $\Delta_{i}$ the relative weight of a given relaxation type, and $\tau_{i}$ is the respective time constant. $\Delta_{1}$ and $\tau_{1}$ are the relative weight and time constant associated with the orientational motion of the biomolecule. For a typical protein solution such as the myoglobin-water system, $\tau_{1}$ is about $74 \mathrm{~ns}^{5,8}\left(\Delta_{2}, \tau_{2}\right)$ and $\left(\Delta_{3}, \tau_{3}\right)$ correspond to the relaxation of biological water associated with the protein. Although these two relaxation phenomena are quite different, they have approximately equal weights. The relaxation times are about $\tau_{2}=10 \mathrm{~ns}$ and $\tau_{3}=$ $40 \mathrm{ps}$, respectively. This behavior is nearly universal and is typically referred to as the bimodality of the reorientational response of biological water. In Table 1, we show a few examples of the bimodal nature of reorientational dynamics of biological water as observed by dielectric relaxation and NMR studies. Finally, $\Delta_{4}$ corresponds to the relative weight of the rotational relaxation of bulk water, and $\tau_{4}$ is the corresponding relaxation time equal to $8.3 \mathrm{ps}$. This bimodal behavior is typical and has been observed with DNA, with water enclosed within the cavities of cyclodextrin, and also in the aqueous medium of reverse micelles.

Many workers have reviewed the results of dielectric measurements on protein-water systems. ${ }^{1-3}$ The earliest measurement of the dielectric properties of protein-water system was made by $\mathrm{Oncley}^{6}$ who concluded that the carboxyhemoglobin molecule is associated with a rotational relaxation time constant of $84 \mathrm{~ns}$. Later, Buchanan et al. ${ }^{7 \mathrm{a}}$ and Haggis et al. ${ }^{7 \mathrm{~b}}$ carried out measurements at higher frequencies and found that about one-third of the total hydration sphere is bound tightly to the water molecule and does not contribute to the dielectric dispersion. The most detailed characterization of a proteinwater solution has been provided by Grant et al. ${ }^{8}$ who showed 
TABLE 1: Examples of the Observed Relaxation Times for Water Associated with Several Biomolecules Probed by Dielectric Relaxation (DR) and NMR Methods

\begin{tabular}{|c|c|c|c|}
\hline $\begin{array}{l}\text { exptl } \\
\text { method }\end{array}$ & $\begin{array}{l}\text { system } \\
\text { studied }\end{array}$ & $\begin{array}{l}\text { relaxation times } \\
\text { observed for water }\end{array}$ & refs \\
\hline DR & $\begin{array}{l}\text { myoglobin } \\
\text { solution }\end{array}$ & $\sim 10 \mathrm{~ns}, \sim 40 \mathrm{ps}$ & Grant et al. ${ }^{8}$ \\
\hline DR & $\begin{array}{l}\text { lysozyme } \\
\text { powder }\end{array}$ & $\sim 1 \mathrm{us}, \sim 20 \mathrm{ps}$ & Harvey et al. ${ }^{11}$ \\
\hline NMR & $\begin{array}{l}\text { apotransferin } \\
\text { solution }\end{array}$ & $\sim 15 \mathrm{~ns}$ & Koenig et al. ${ }^{27}$ \\
\hline NMR & $\begin{array}{l}\text { hemoglobin } \\
\text { solution }\end{array}$ & $\sim 15 \mathrm{~ns}$ & Koenig et al. ${ }^{27}$ \\
\hline DR & $\begin{array}{l}\text { metmyoglobin } \\
\text { powder }\end{array}$ & $\sim 10 \mathrm{~ns}$ & Singh et al. ${ }^{3}$ \\
\hline DR & $\begin{array}{l}\text { D.NA. } \\
\text { solution }\end{array}$ & $\sim 1 \mathrm{us}, \sim 10 \mathrm{ps}$ & Mashimo et al. ${ }^{14}$ \\
\hline DR & 18 biomaterials & $1 \mathrm{us}, \sim 10 \mathrm{ps}$ & Mashimo et al. ${ }^{14}$ \\
\hline NMR & $\begin{array}{l}\text { lysozyme } \\
\text { powder }\end{array}$ & $\sim 1 \mathrm{us}, \sim 10 \mathrm{ps}$ & Fullerton et al. ${ }^{6}$ \\
\hline NMR & $\begin{array}{l}\text { lysozyme } \\
\text { powder }\end{array}$ & $\sim 1 \mathrm{us}, \sim 100 \mathrm{ps}$ & Peemoeller et al. ${ }^{18}$ \\
\hline NMR & $\begin{array}{l}\text { crambin } \\
\text { powder }\end{array}$ & $320 \mathrm{ps}$, & Usha et al. ${ }^{19}$ \\
\hline NMR & $\begin{array}{l}\text { several } \\
\text { proteins }\end{array}$ & $\sim 10 \mathrm{~ns}, \sim 20 \mathrm{ps}$ & Halle et al. ${ }^{26}$ \\
\hline
\end{tabular}

that protein-bound water exhibits a range of relaxation time constants. They concluded that these time constants are associated with distinct processes such as the rotational motion of the protein and of biological and bulk water. Studies of Schawan and coworkers, ${ }^{9}$ of Takashima and co-workers, ${ }^{10}$ and of Hoekstra ${ }^{11}$ also revealed distinct relaxation times of biological water and confirmed the results of Grant et al. Pethig and coworkers ${ }^{5}$ concluded that the primary hydration layer is strongly bound and rotationally hindered and the microwave dielectric behavior is predominantly influenced by thermally activated water in the secondary layer of biological water. Several other studies ${ }^{12-19}$ have addressed the origin of the distinct relaxation times observed in microwave experiments.

Computer simulations can provide useful insight into the structure and the energetics of the solvent. ${ }^{20}$ On the basis of Monte Carlo simulation of small peptides and proteins, it has been concluded that water molecules far from the protein move over larger distances and are more ordered than those near it. Many of the water molecules close to the protein are considerably lower in energy than those of bulk water.

Two completely different schools of thought exist regarding the existence of the two distinct time scales. The first approach advocates restricted slow orientational relaxation of partly bound water molecules. Yet, it is known that the concentration of the partly bound, singly hydrogen-bonded water molecules within the hydration shell in the immediate vicinity of the biomolecule is low. Mashimo et al. ${ }^{14}$ pointed out that if the single hydrogenbonded species were present to a large extent, the variation in the magnitude of the slow relaxation time constant would have been much larger than that observed experimentally. Moreover, the low-frequency absorption is of pure a Debye type, which also excludes the existence of two or more polar species. Koenig et al. ${ }^{27}$ pointed out that the inclusion of the partly bound species in the theoretical model does not help to better understand the results of the NMR observations of protein solution. Therefore, the explanation of the bimodal dielectric response in terms of the partly bound water molecules is controversial.

The alternative explanation is based on assuming a dynamic equilibrium between the free and bound water. However, this approach has not been developed to treat the dielectric relaxation quantitatively, which is somewhat surprising given the importance of the problem.

The objective of this article is to present a quantitative theory of dielectric relaxation of biological water based on a dynamic equilibrium between the free and bound water molecules. The theory is based on the assumption that a dynamic exchange between the free and bound water states, controlled by the strength and the number of hydrogen bond(s) between the water molecule and the biomolecule, determines the observed dynamics. Since the dynamics of the rigidly bound water is coupled to the biomolecular rotation, ${ }^{1-3}$ this aspect has also been included in our study.

Our results are found to be in very good agreement with the known experimental results. A merit of the present explanation is that it is based on a molecular picture and that it provides quantitative explanation of the presence of two widely separated time scales.

The organization of the rest of the paper is as follows. In the section II we describe the dynamical model of bound and free water. In the section III we present the theoretical formulation and describe the equation of motion governing the dynamical equilibrium between bound and free water. Section IV includes the calculation of the frequency dependent dielectric function of biological water from the moment-moment correlation function, and in section $\mathrm{V}$ we present the ingredients necessary for the theoretical calculation. In the section VI we discuss the results. In the section VII we present a scheme of calculation of the frequency dependent dielectric function of a protein solution. This is followed by brief concluding remarks.

\section{Dynamical Model of Free and Bound Water Near a Biomolecule}

In this section we shall first discuss the essential features of our model. We term the water molecules enclosed within the solvation shell present in the immediate vicinity of the biomolecule as "biological water". This "biological water" comprises two components: one is "free water" and the other is attached to the biomolecule by a strong hydrogen bond and rotates only in a coupled fashion with the slowly rotating biomolecule. The latter species is termed "bound water". Beyond this solvation shell the water molecules behave as ordinary water, and we term them "bulk water". A pictorial description of the model is given in Figure 1. Thus, our model consists of three parts. First, it is assumed that the "free water" molecules are free to rotate and to contribute to the dielectric relaxation process. The bound water are at least doubly hydrogen bonded, so they can rotate only in concert with the biomolecule. Second, the following dynamic exchange between the free and bound water species exists at all times:

$$
\left[\mathrm{H}_{2} \mathrm{O}\right]_{\text {free state }} \rightleftharpoons\left[\mathrm{H}_{2} \mathrm{O}\right]_{\text {bound state }}
$$

The equilibrium constant for the dynamical equilibrium between the free and bound water can be written as follows:

$$
K=\frac{k_{2}}{k_{1}}=\mathrm{e}^{-\Delta G^{0} /(R T)}
$$

where $\Delta G^{0}$ is the difference in the hydrogen bond free energy, per mole, of a water molecule, between a bond to a biopolymer and to a free water molecule. ${ }^{4 a}$ Figure 1 illustrates schematically the dynamic exchange between the bound and free water species at a globular protein surface. $k_{1}$ and $k_{2}$ are the rate constants of the exchange between free and bound states. Third, the effect of the rotation of the biomolecule is included in the theoretical scheme. 


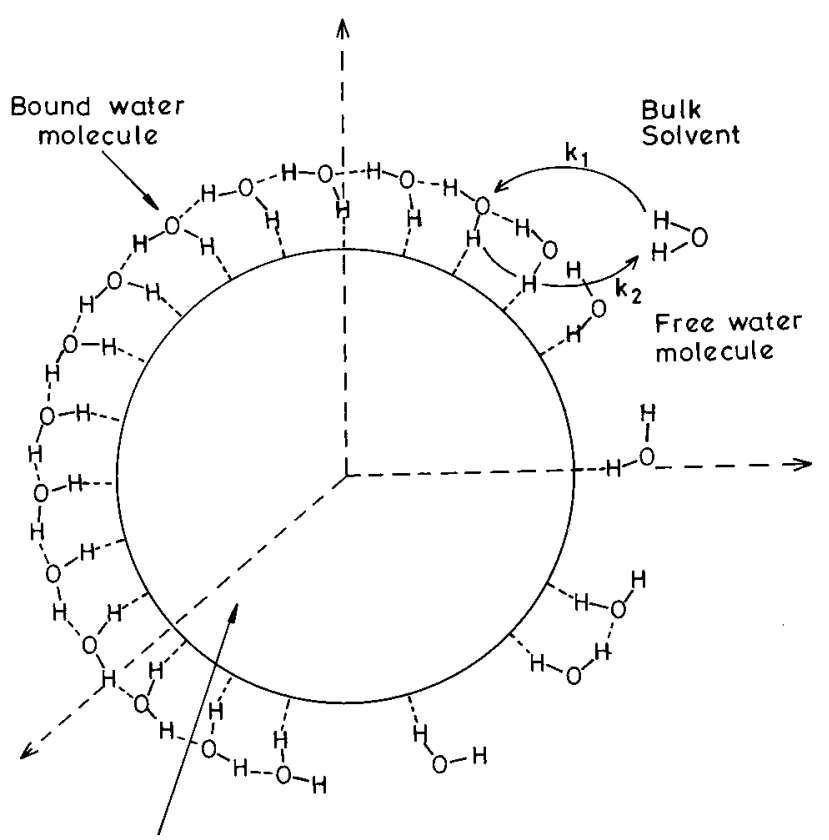

A Spherical biomolecule

Figure 1. Schematic representation of the dynamic exchange between bound and free water species at the globular protein surface. $k_{1}$ and $k_{2}$ are the rate constants for exchange between free and bound state. Only very few water molecules are single hydrogen bonded. The bound and free water molecules are the two components of the "biological water".

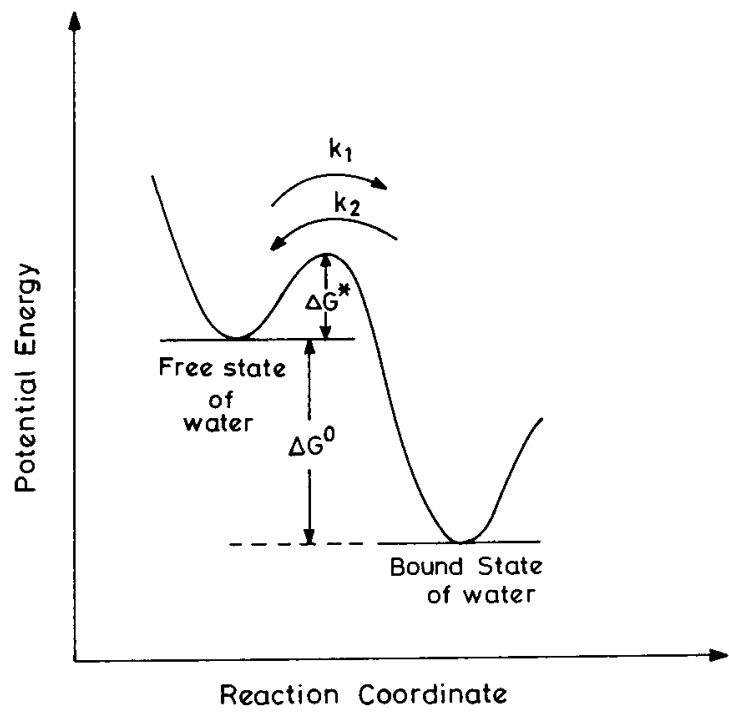

Figure 2. Schematic potential energy diagram showing the free water state, bound water state, and activated complex for the dynamic equilibrium $\left[\mathrm{H}_{2} \mathrm{O}\right]_{\text {free state }} \rightleftharpoons\left[\mathrm{H}_{2} \mathrm{O}\right]_{\text {bound state }}$ versus the reaction coordinate. The $\Delta G^{0}$ is the difference in the free energy of a hydrogen bond of a water molecule between a biomolecule and a solvent water. ${ }^{32}$ The $\Delta G^{*}$ is the energy of activation.

The orientations of the free and bound water molecule are given by $\Omega_{\mathbf{f}}$ and $\Omega_{\mathbf{b}}$, respectively. We introduce two time dependent densities, $\rho_{\mathrm{f}}\left(\Omega_{\mathrm{f}}, t\right)$ and $\rho_{\mathrm{f}}\left(\Omega_{\mathrm{b}}, t\right)$ to describe the fluctuating densities of the free and bound water molecules in the hydration shell. Note that the orientation of the bound state is determined by the orientation of the biomolecule itself because of its coupling to the biomolecule by hydrogen bonds (see Figure 1). In Figure 2, we have depicted a schematic potential energy profile for the dynamic equilibrium between the bound and free water states. The bound water molecules are in a lower energy state compared to their free state. This energy difference is denoted by $\Delta G^{0}$. This energy difference is determined by the strength and the number of hydrogen bonds. The activation energy of desorption from the surface of the biomolecule is denoted by $\Delta G^{*}$.

\section{Theoretical Formulation}

The following general diffusion equations feature the coupled dynamic behavior of free and bound water molecules

$$
\begin{aligned}
& \frac{\partial \rho_{\mathrm{f}}\left(\Omega_{\mathbf{f}}, t\right)}{\partial t}= \\
& D_{\mathrm{R}}^{\mathrm{W}} \nabla_{\Omega_{\mathrm{f}}}{ }^{2} \rho_{\mathrm{f}}\left(\Omega_{\mathbf{f}}, t\right)-\rho_{\mathrm{f}}\left(\Omega_{\mathbf{f}}, t\right) \int k_{1}\left(\Omega_{\mathbf{f}} \rightarrow \Omega_{\mathbf{b}}\right) \mathrm{d} \Omega_{\mathbf{b}}+ \\
& \int k_{2}\left(\Omega_{\mathbf{b}} \rightarrow \Omega_{\mathrm{f}}\right) \rho_{\mathrm{b}}\left(\Omega_{\mathbf{b}}, t\right) \mathrm{d} \Omega_{\mathbf{b}} \\
& \frac{\partial \rho_{\mathrm{b}}\left(\Omega_{\mathbf{b}}, t\right)}{\partial t}=-\rho_{\mathrm{b}}\left(\Omega_{\mathbf{b}}, t\right) \int k_{2}\left(\Omega_{\mathbf{b}} \rightarrow \Omega_{\mathbf{f}}\right) \mathrm{d} \Omega_{\mathbf{f}}+ \\
& \int k_{1}\left(\Omega_{\mathbf{f}} \rightarrow \Omega_{\mathbf{b}}\right) \rho_{\mathrm{f}}\left(\Omega_{\mathbf{f}}, t\right) \mathrm{d} \Omega_{\mathbf{f}}+D_{\mathrm{R}}^{\mathrm{B}} \nabla_{\Omega_{\mathrm{b}}}{ }^{2} \rho_{\mathrm{b}}\left(\Omega_{\mathbf{b}}, t\right)
\end{aligned}
$$

where $D_{\mathrm{R}}^{\mathrm{W}}$ is the rotational diffusion constant of the free water molecule and the $D_{\mathrm{R}}^{\mathrm{B}}$ is the rotational diffusion constant of the biomolecule. Note that here "free" and "bound" refer to the two constituents of the biological water.

To proceed further we assume that the orientation of the molecule does not change during the free $\leftrightarrow$ bound reaction (see eq 1). That is, we assume that the rate constants $k_{1}\left(\Omega_{\mathbf{f}} \rightarrow\right.$ $\left.\Omega_{\mathbf{b}}\right)$ and $k_{2}\left(\Omega_{\mathbf{b}} \rightarrow \Omega_{\mathbf{f}}\right)$ are local and can thus be approximated by the respective $\delta$ functions

$$
\begin{aligned}
& k_{1}\left(\Omega_{\mathbf{f}} \rightarrow \Omega_{\mathbf{b}}\right)=\delta\left(\Omega_{\mathbf{f}}-\Omega_{\mathbf{b}}\right) \\
& k_{2}\left(\Omega_{\mathbf{b}} \rightarrow \Omega_{\mathbf{f}}\right)=\delta\left(\Omega_{\mathbf{b}}-\Omega_{\mathbf{f}}\right)
\end{aligned}
$$

We assume that the shape of the biomolecule is spherical and $D_{\mathrm{R}}^{\mathrm{B}}$ is isotropic. The above equations are then simplified as follows:

$$
\begin{aligned}
\frac{\partial \rho_{\mathrm{f}}\left(\Omega_{\mathrm{f}}, t\right)}{\partial t}=D_{\mathrm{R}}^{\mathrm{W}} \nabla_{\Omega_{\mathrm{f}}}^{2} \rho_{\mathrm{f}}\left(\Omega_{\mathrm{f}}, t\right)-k_{1} \rho_{\mathrm{f}}\left(\Omega_{\mathrm{f}}, t\right)+k_{2} \rho_{\mathrm{b}}\left(\Omega_{\mathrm{f}}, t\right) \\
\frac{\partial \rho_{\mathrm{b}}\left(\Omega_{\mathbf{b}}, t\right)}{\partial t}=-k_{2} \rho_{\mathrm{b}}\left(\Omega_{\mathbf{b}}, t\right)+k_{1} \rho_{\mathrm{f}}\left(\Omega_{\mathbf{b}}, t\right)+ \\
D_{\mathrm{R}}^{\mathrm{B}} \nabla_{\Omega_{\mathrm{b}}}{ }^{2} \rho_{\mathrm{b}}\left(\Omega_{\mathrm{b}}, t\right)
\end{aligned}
$$

From the eqs 6 and 7 we can easily derive the following two differential equations for the free and bound water species:

$$
\begin{aligned}
& \frac{\partial^{2} \rho_{\mathrm{f}}\left(\Omega_{\mathrm{f}}, t\right)}{\partial t^{2}}+\left[l(l+1) D_{\mathrm{R}}^{\mathrm{W}}+k_{1}+k_{2}+l(l+\right. \\
& \text { 1) } \left.D_{\mathrm{R}}^{\mathrm{B}}\right] \frac{\partial \rho_{\mathrm{f}}\left(\Omega_{\mathrm{f}}, t\right)}{\partial t}=+\left[l(l+1) k_{2} D_{\mathrm{R}}^{\mathrm{W}}+l^{2}(l+1)^{2} D_{\mathrm{R}}^{\mathrm{B}} D_{\mathrm{R}}^{\mathrm{W}}+\right. \\
& \left.k_{1} l(l+1) D_{\mathrm{R}}^{\mathrm{B}}\right] \rho_{\mathrm{f}}\left(\Omega_{\mathrm{f}}, t\right)=0 \\
& \frac{\partial^{2} \rho_{\mathrm{b}}\left(\Omega_{\mathbf{b}}, t\right)}{\partial t^{2}}+\left[k_{1}+k_{2}+l(l+1) D_{\mathrm{R}}^{\mathrm{B}}\right] \frac{\partial \rho_{\mathrm{b}}\left(\Omega_{\mathbf{b}}, t\right)}{\partial t}+ \\
& l(l+1) k_{1} D_{\mathrm{R}}^{\mathrm{B}} \rho_{\mathrm{b}}\left(\Omega_{\mathrm{b}} t\right)=0
\end{aligned}
$$

The orientation and time dependent density, $\rho_{\mathrm{f}}\left(\Omega_{\mathbf{f}}, t\right)$ can be expanded in terms of spherical harmonics as follows:

$$
\rho_{\mathrm{f}}\left(\Omega_{\mathbf{f}}, t\right)=\sum_{l m} a_{l m}^{\mathrm{f}}(t) Y_{l m}\left(\Omega_{\mathbf{f}}\right)
$$


where $a_{l m}^{\mathrm{f}}(t)$ are the expansion coefficients and the $Y_{l m}\left(\Omega_{\mathbf{f}}\right)$ are the spherical harmonics of rank $l$ and projection $m$.

Thus, we have a solution of the form where the $C_{1}$ and $C_{2}$

$$
a_{l m}^{\mathrm{f}}(t)=\left[C_{1} \mathrm{e}^{-n_{1}^{l} t}+C_{2} \mathrm{e}^{-n_{2}{ }^{t}}\right]
$$

are the coefficients of two relaxation modes. $n_{1}$ and $n_{2}$ have the following forms:

$$
\begin{aligned}
& n_{1}^{l}=-\frac{-X-\sqrt{X^{2}-4 Y}}{2} \\
& n_{2}^{l}=-\frac{-X+\sqrt{X^{2}-4 Y}}{2}
\end{aligned}
$$

where

$$
X=\left[l(l+1) D_{\mathrm{R}}^{\mathrm{W}}+k_{1}+k_{2}+l(l+1) D_{\mathrm{B}}^{\mathrm{R}}\right]
$$

and

$$
Y=\left[l(l+1) D_{\mathrm{R}}^{\mathrm{W}} k_{2}+l^{2}(l+1)^{2} D_{\mathrm{R}}^{\mathrm{W}} D_{\mathrm{B}}^{\mathrm{R}}+l(l+1) k_{1} D_{\mathrm{B}}^{\mathrm{R}}\right]
$$

Similarly, for the bound water species, we have a solution of the form

$$
a_{l m}^{\mathrm{b}}(t)=\left[C_{3} \mathrm{e}^{-n_{3} t}+C_{4} \mathrm{e}^{-n_{4} l^{t}}\right]
$$

where the $C_{3}$ and $C_{4}$ are the coefficients of the two relaxation modes. $n_{3}^{l}$ and $n_{4}^{l}$ have the following forms:

$$
\begin{aligned}
& n_{3}^{l}=-\frac{-M-\sqrt{M^{2}-4 N}}{2} \\
& n_{4}^{l}=-\frac{-M+\sqrt{M^{2}-4 N}}{2}
\end{aligned}
$$

where

$$
M=\left[k_{1}+k_{2}+l(l+1) D_{\mathrm{R}}^{\mathrm{B}}\right]
$$

and

$$
N=\left[l(l+1) k_{1} D_{\mathrm{R}}^{\mathrm{B}}\right]
$$

For a typical protein $D_{\mathrm{R}}^{\mathrm{B}}$ is very small. For example, for myoglobin, $D_{\mathrm{R}}^{\mathrm{B}}$ is about $10^{7} \mathrm{~s}^{-1}$, while the rate constants $k_{1}$ and $k_{2}$ are on the order of $10^{10}-10^{11} \mathrm{~s}^{-1}$. Thus, $a_{l m}^{\mathrm{b}}(t)$ can be written as follows:

$$
a_{l m}^{\mathrm{b}}(t)=\left[C_{3} \mathrm{e}^{-n_{3} t}+C_{4}\right]
$$

We evaluate the constants $C_{1}, C_{2}, C_{3}$, and $C_{4}$ from the following relations. At $t=0$ we obtain

$$
C_{1}+C_{2}=1=C_{3}+C_{4}
$$

At $t=\infty$, we obtain $a_{l m}^{\mathrm{b}}(t)=0$. Consequently,

$$
C_{3}=1, \quad C_{4}=0
$$

From the equation of conservation of densities for free and bound species we obtain

$$
\left\langle\rho_{\mathrm{f}}\right\rangle\left[C_{1} n_{1}+C_{2} n_{2}\right]+\left\langle\rho_{\mathrm{b}}\right\rangle\left[C_{3} n_{3}\right]=0
$$

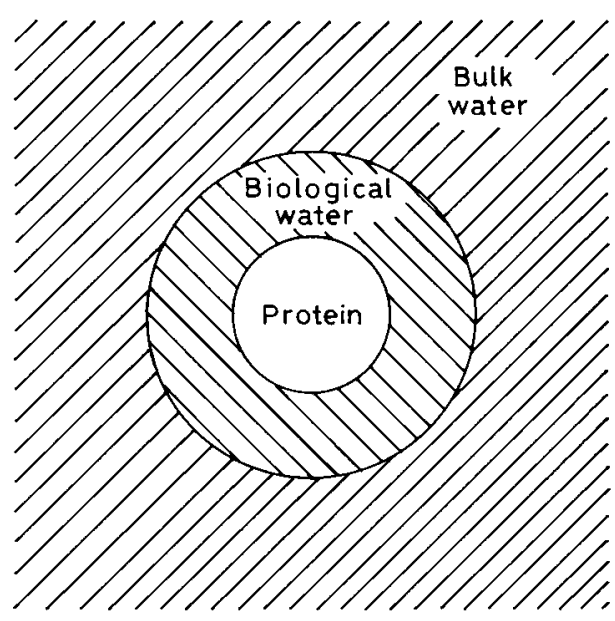

Figure 3. Schematic representation of the shell of biological water and bulk water for which the frequency dependent dielectric function are calculated in section IV. The Cole-Cole plots and the plot of $\epsilon^{\prime}$ against frequency are shown in Figures 5 and 6, respectively.

Here, we have approximated $\rho_{\mathrm{f}}(t=0)=\left\langle\rho_{\mathrm{f}}\right\rangle$ and $\rho_{\mathrm{b}}(t=0)=$ $\left\langle\rho_{\mathrm{b}}\right\rangle$, where the $\left\langle\rho_{\mathrm{f}}\right\rangle$ and $\left\langle\rho_{\mathrm{b}}\right\rangle$ are the average number densities of the free and bound species, respectively.

We can evaluate the constants from the above relations to obtain

$$
a_{l m}^{\mathrm{f}}(t)=\left[C_{1} \mathrm{e}^{-n_{1}^{l} t}+C_{2} \mathrm{e}^{-n_{2} t t}\right]
$$

and

$$
a_{l m}^{\mathrm{b}}(t)=\left[\mathrm{e}^{-n_{3} t}\right]
$$

\section{Calculation of the Frequency Dependent Dielectric Function of Biological Water}

For a system composed of bound and free water molecules, the moment-moment correlation function of the system can be written as follows:

$$
C_{\mathrm{MM}}(t)=N^{2}\left[\left(\frac{N_{\mathrm{f}}}{N}\right)^{2}\left\langle\vec{\mu}_{\mathrm{f}}(t) \vec{\mu}_{\mathrm{f}}(0)\right\rangle+\left(\frac{N_{\mathrm{b}}}{N}\right)^{2}\left\langle\vec{\mu}_{\mathrm{b}}(t) \vec{\mu}_{\mathrm{b}}(0)\right\rangle\right]
$$

where the orientation and time dependent dipole moment for the free water molecules is $\vec{\mu}_{\mathrm{f}}$ and that for bound water $\vec{\mu}_{\mathrm{b}} . N_{\mathrm{f}}$ and $N_{\mathrm{b}}$ are the number of free and bound water molecules per mole $\left(N_{\mathrm{f}}+N_{\mathrm{b}}=N\right)$. Thus,

$$
\begin{aligned}
C_{\mathrm{MM}}(t) & =N^{2} \mu^{2}\left[n_{\mathrm{f}}^{2}\left(C_{1} \mathrm{e}^{-n_{1} t}+C_{2} \mathrm{e}^{-n_{2} t}\right)+n_{\mathrm{b}}{ }^{2}\left(\mathrm{e}^{-n_{3} t}\right)\left(\mathrm{e}^{-t / \tau \mathrm{R}}\right)\right] \\
& =N^{2} \mu^{2}\left[\left(w_{1} \mathrm{e}^{-n_{1}^{l} t}+w_{2} \mathrm{e}^{-n_{2} t^{t}}\right)+w_{3} \mathrm{e}^{-n_{3} t}\left(\mathrm{e}^{-t / \tau_{\mathrm{R}}^{\mathrm{B}}}\right)\right]
\end{aligned}
$$

where $n_{\mathrm{f}}$ and $n_{\mathrm{b}}$ are the respective number fractions of free and bound species. $w_{1}=n_{\mathrm{f}}^{2} C_{1}, w_{2}=n_{\mathrm{f}}^{2} C_{2}$, and $w_{3}=n_{\mathrm{b}}^{2}$ are the relative weights of the relaxation modes.

For simplicity we assume that the biomolecule is spherical, having a radius $R_{\mathrm{p}}$. The biomolecule is surrounded by a solvation shell, which is composed of biological water. This is also assumed to be of spherical shape. This geometry is depicted in Figure 3. The relaxation behavior of the hydration shell is characterized by a frequency dependent dielectric function $\epsilon^{\mathrm{B}}(\omega)$. The value of the dielectric function at zero and infinite frequencie are denoted by $\epsilon_{\mathrm{s}}^{\mathrm{B}}$ and $\epsilon_{\infty}^{\mathrm{B}}$, respectively. Beyond this solvation shell the water molecules behave as ordinary water and are termed bulk water. In the present study, we assume that the bulk water has a frequency dependent 
dielectric function $\epsilon^{\mathrm{W}}(\omega)$. Similarly, the value of the dielectric function at zero and infinite frequency are denoted by $\epsilon_{\mathrm{s}}^{\mathrm{W}}$ and $\epsilon_{\infty}^{\mathrm{W}}$, respectively. $\epsilon^{\mathrm{W}}(\omega)$ is characterized by a Debye type relaxation with time constant $\tau_{\mathrm{D}}$. All of these parameters have values known for bulk water.

For uniform polarization geometries, the net dipole moment per unit volume of the hydration shell resulting from an external field with frequency $\omega$ is related to $\epsilon_{\mathrm{s}}^{\mathrm{B}}$ and the macroscopic electric field inside the shell $E_{\mathrm{z}}(t)$ through the following relation. ${ }^{29}$

$$
\left\langle m_{\mathrm{z}}(t)\right\rangle_{E(\infty)}=\frac{\left[\epsilon^{\mathrm{B}}(\omega)-\epsilon_{\infty}^{\mathrm{B}}\right]}{4 \pi} V_{\text {shell }} E_{z}(t)
$$

where $V_{\text {shell }}$ is the volume of the hydration shell. Again, we can derive $\left\langle m_{z}(t)\right\rangle_{E(\infty)}$ from the autocorrelation function of the net dipole moment in the absence of the field as follows:

$$
\left\langle m_{\mathrm{z}}(t)\right\rangle_{E(\infty)}=-\frac{1}{k_{\mathrm{B}} T} \int_{0}^{\infty} E_{z}^{\mathrm{c}}\left(t-t^{\prime}\right) \frac{\mathrm{d}}{\mathrm{d} t}\left\langle m_{z}(0) m_{z}\left(t^{\prime}\right)\right\rangle \mathrm{d} t^{\prime}
$$

The cavity field of the hydration shell is obtained from the electrostatic boundary value calculations ${ }^{30}$ as follows:

$$
E_{z}^{\mathrm{c}}(t)=\frac{3 \epsilon^{\mathrm{W}}(\omega)}{2 \epsilon^{\mathrm{W}}(\omega)+\epsilon_{\infty}^{\mathrm{W}}} E(\infty)(t)
$$

$E_{z}(t)$ is related to $E(\infty)(t)$ by the following relation:

$$
E_{z}(t)=\frac{3 \epsilon^{\mathrm{W}}(\omega)}{2 \epsilon^{\mathrm{W}}(\omega)+\epsilon^{\mathrm{B}}(\omega)} E(\infty)(t)
$$

On the basis of these equations, we can write the following relation between the normalized moment - moment correlation function $\phi(t)$ and the $\epsilon^{\mathrm{B}}(\omega)$ :

$$
\mathrm{L}\left[-\frac{\mathrm{d}}{\mathrm{d} t} \phi(t)\right]=\frac{\left[\epsilon^{\mathrm{B}}(\omega)-\epsilon_{\infty}^{\mathrm{B}}\right]\left[2 \epsilon^{\mathrm{W}}(\omega)+\epsilon_{\infty}^{\mathrm{W}}\right]\left[2 \epsilon_{s}^{\mathrm{W}}-\epsilon_{s}^{\mathrm{B}}\right]}{\left[2 \epsilon^{\mathrm{W}}(\omega)+\epsilon^{\mathrm{B}}(\omega)\right]\left[\epsilon_{s}^{\mathrm{B}}-\epsilon_{\infty}^{\mathrm{B}}\right]\left[2 \epsilon_{s}^{\mathrm{W}}+\epsilon_{\infty}^{\mathrm{W}}\right]}
$$

where $L$ denotes the Laplace transform, with the Laplace transform variable $z=\mathrm{i} \omega$. This is the same general relation derived by Titulaer and Deutch many years ago. ${ }^{29}$ Next, we use eq 33 to calculate the frequency dependent dielectric function of biological water.

\section{Calculation of the Rate Constants and the Choice of the Hydrogen Bond Strength Necessary for Theoretical Calculation}

In this subsection we discuss the calculation and/or determination of the parameters, i.e., the rate constants $k_{1}$ and $k_{2}$ and other parameters such as the rotational diffusion coefficient $\left(D_{\mathrm{R}}^{\mathrm{W}}\right)$, the excess hydrogen bond energy of water with the biomolecule (over that in the bulk) $\Delta G^{0}$, the activation energy $\Delta G^{*}$ (see Figure 2), and the mole fraction for bound and free water $m_{\mathrm{b}}$ and $m_{\mathrm{f}}$, respectively.

From the activated complex theory, ${ }^{31}$ we have

$$
k_{1} \simeq \frac{k_{\mathrm{B}} T}{h} \mathrm{e}^{-\Delta G^{\ddagger} /(R T)}
$$

where is the $\Delta G^{\ddagger}=\left(\Delta G^{*}+\Delta G^{0}\right), k_{\mathrm{B}}$ the Boltzmann constant, $h$ the Planck's constant, $R$ the universal gas constant, and $T$ the temperature. By use of the two equations given above, the rate constants $k_{1}$ and $k_{2}$ can be easily calculated.
TABLE 2: Parameters Necessary for the Calculation of the Dielectric Relaxation Parameters from Microscopic Theory, Calculated Rate Constants for the Dynamic Exchange between the Free and Bound Water $\left(k_{1}\right.$ and $\left.k_{2}\right)$, and Dielectric Relaxation Times for Slow and Fast Relaxation Processes $\left(\tau_{1} \text { and } \tau_{3}\right)^{a}$

\begin{tabular}{ccccc}
\hline$\Delta G^{\circ}$ & $k_{1} \times 10^{-12}\left(\mathrm{~s}^{-1}\right)$ & $k_{2} \times 10^{-12}\left(\mathrm{~s}^{-1}\right)$ & $\tau_{1} \times 10^{12}(\mathrm{~s})$ & $\tau_{3} \times 10^{12}(\mathrm{~s})$ \\
\hline-1.4 & 0.13 & $0.3 \times 10^{-2}$ & $4.82 \times 10^{2}$ & 15.17 \\
-2.0 & 0.05 & $0.4 \times 10^{-3}$ & $3.15 \times 10^{3}$ & $4.25 \times 10^{1}$ \\
-2.8 & 0.01 & $0.23 \times 10^{-4}$ & $4.41 \times 10^{4}$ & $1.66 \times 10^{2}$ \\
-4.0 & $0.15 \times 10^{-2}$ & $0.4 \times 10^{-6}$ & $2.52 \times 10^{6}$ & $1.26 \times 10^{3}$
\end{tabular}

${ }^{a}$ In each case, the number fraction for bound water and free water is 0.5 and 0.5 , respectively. $\Delta G^{*}$ is $1.5 k_{\mathrm{B}} T$ in all the cases.

The rotational diffusion constant for the free water near the biomolecule is approximated by the bulk value. The rotational diffusion coefficient of free water $D_{\mathrm{R}}^{\mathrm{W}}$ near the biomolecule may be somewhat different from the bulk value. It has been concluded that at the macromolecular surface, the solvent motion is only slightly modified by the protein. ${ }^{23}$ The self-diffusion coefficient of water at the surface of lysozyme is reduced by less than a factor of 10 at distances closer than $10 \AA$ from the surface. $^{23}$ However, to our knowledge, there is no report of the $D_{\mathrm{R}}^{\mathrm{W}}$ value of water near a biomolecule, and we have taken it to be same as that of bulk $\left(0.22 \times 10^{12} \mathrm{~s}^{-1}\right)$.

From the dielectric relaxation measurements of different protein solutions ${ }^{5 \mathrm{a}, 8 \mathrm{k}}$ it is known that two nearly equal populations of total protein hydration are responsible for the slow (in nanoseconds) and fast (in picoseconds) relaxation modes of biological water. However, owing to the stabilization by the hydrogen bond, the bound water molecules are expected to be more abundant than the free water species. In the present study, we have taken the mole fraction of bound and free water as 0.5 and 0.5 , respectively.

The energy per hydrogen bond can vary widely from one biomolecule to another. For example, different peptide groups are associated with hydrogen bond energies, ranging from -1.0 to $-1.4 \mathrm{kcal} / \mathrm{mol}$ for polar, uncharged groups and from -1.5 to $-2.8 \mathrm{kcal} / \mathrm{mol}$ for charged groups. ${ }^{32}$ According to the model proposed by Mashimo et al., ${ }^{14}$ a bound water molecule is engaged in two hydrogen bonds. One is a $[\mathrm{O}-\mathrm{H} \cdots \mathrm{O}]$ bond, which forms a water multimer, and the other hydrogen bond is engaged in binding the water to the biomolecule. For the bound water molecules to be rotationally free, both these two bonds have to be broken. The total energy of these two bonds is the activation energy that has to be overcome in order to give full orientational freedom to the water molecule. In the present study we have shown results for dielectric relaxation, considering the total hydrogen bond energy as varying over a wide range.

\section{Numerical Results}

In the Table 2 we present the calculated values of the rate constants associated with the dynamic equilibrium between free and bound water, the relative weights, and the time constants for the slow and fast relaxation processes. In the first column the values for the total hydrogen bond strength of the doubly bound water molecules are given. As pointed out in section $\mathrm{V}$, different peptide groups have energy per hydrogen bond ranging approximately from -1.0 to $-2.8 \mathrm{kcal} \mathrm{mol}^{-1}$. We have varied the magnitude of $\Delta G^{0}$ as input from -1.4 to $-4.0 \mathrm{kcal}$ $\mathrm{mol}^{-1}$. Note that the two hydrogen bonds attached with the bound water molecule may have different energies because one is attached to the biomolecule and the other to the neighboring water in the hydration shell. We need to consider the total bondbreaking energy of two bonds. 


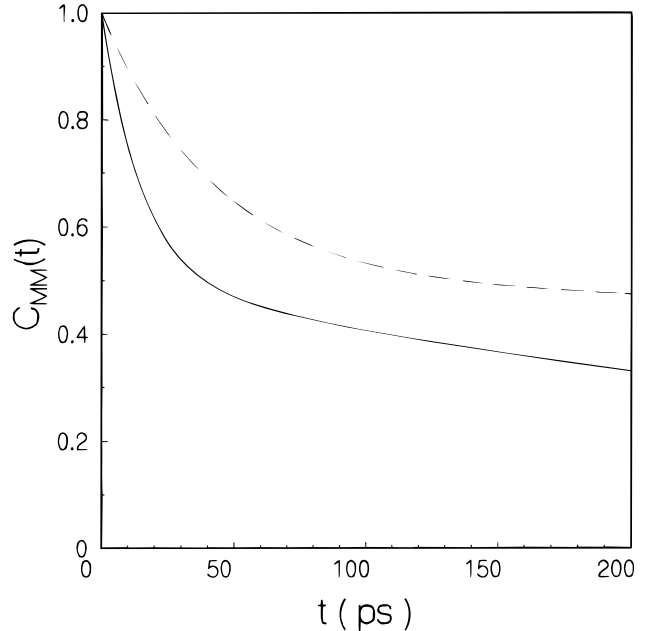

Figure 4. Plot of normalized $C_{\mathrm{MM}}(t)$ against $t$ (in ps) for different hydrogen bond energies involving biological water (for details see section IV). The solid line corresponds to a total hydrogen bond energy of $-1.4 \mathrm{kcal} / \mathrm{mol}$, and the dashed line corresponds to a total hydrogen bond energy of $-2.0 \mathrm{kcal} / \mathrm{mol}$. The mole fractions for bound and free water are 0.5 and 0.5 , respectively, for both plots. $\Delta G^{*}=1.5 k_{\mathrm{B}} T$.

It is clearly seen that as the strength of the hydrogen bond increases, the magnitude of the rate constant $k_{1}$ also increases. Naturally, as the strength of the hydrogen bond is increased, the water molecules prefer to exist as bound species. The relaxation time constant of the slow relaxation component therefore increases. At the lowest $\Delta G^{0}$ value $\left(-1.4 \mathrm{kcal} \mathrm{mol}^{-1}\right)$, the relaxation time constant for the slow component is $482 \mathrm{ps}$, while at the highest $\Delta G^{0}$ value $\left(-4.0 \mathrm{kcal} \mathrm{mol}^{-1}\right)$ it becomes $2.5 \mathrm{~ms}$.

Thus, the two-state model presented here indicates that the slow component observed in the dielectric measurements of biomolecules can indeed emerge from a fast dynamic exchange between bound and free water molecules. The strength and the time constant of the slow component are determined by the strength of the hydrogen bonds by which the bound water molecule is attached to the biomolecule and also by the relative stability of the two species.

In Figure 4 we show the results for the normalized momentmoment correlation function $\left(C_{\mathrm{MM}}(t)\right)$ for different hydrogen bond energies. The plot of $C_{\mathrm{MM}}(t)$ versus time shows a strong bimodal decay. The initial relaxation is very fast, in the range of tens of picoseconds, while the later decay occurs on a nanosecond time scale. This bimodal behavior is in excellent agreement with the results reported in the literature. ${ }^{1-3,5-8}$ With increase in the strength of the hydrogen bond, the relaxation becomes increasingly slower, as expected.

Next, we describe the dielectric relaxation of biological water. The Cole-Cole plot is shown in the Figure 5. This also clearly demonstrates the bimodal nature of the relaxation. In Figure 6 we have plotted the real part of the frequency dependent dielectric function and compared it with a typical experimental result. The experimental result associated with the dielectric relaxation of biological water is illustrated by $\delta$ in the experimental graph (see Figure 6a). In the theoretical figure (graph 6b) only this part is shown, with an inset to illustrate the behavior at low frequencies. Note that the agreement between the two is satisfactory. We show in the next section that biomolecular rotation can profoundly influence the dielectric relaxation in the low-frequency (megahertz $(\mathrm{MHz})$ regime, if the biomolecule has a large dipole moment by itself, for example, in lysozyme.

Different residence times in the subnanosecond range have been observed with NMR for surface hydration water molecules

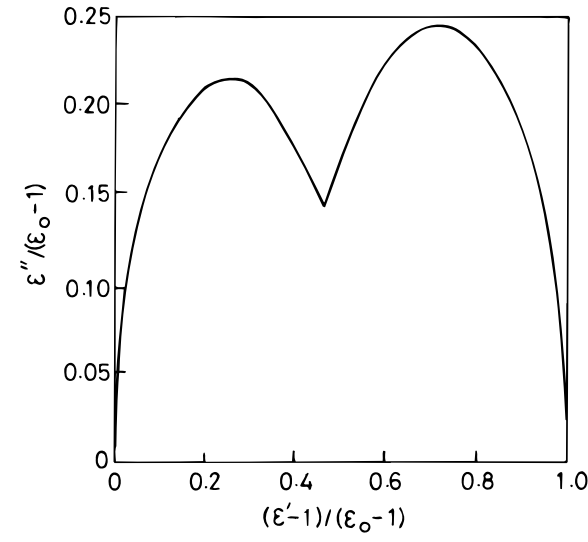

Figure 5. Cole-Cole plot, calculated from the frequency dependent dielectric function of biological water (see section IV for details). The total hydrogen bond energy is $-2.0 \mathrm{kcal} / \mathrm{mol}$. The mole fractions for bound and free water are 0.5 and 0.5 , respectively. The static $\left(\epsilon_{\mathrm{s}}^{\mathrm{B}}\right)$ and infinite $\left(\epsilon_{\infty}^{\mathrm{B}}\right)$ frequency dielectric constant of biological water are taken as 30 and 5.2, respectively. The static and infinite frequency dielectric constant of the bulk water are taken as $\epsilon_{\mathrm{s}}^{\mathrm{W}}=78.36$ and $\epsilon_{\infty}^{\mathrm{W}}=5.2$. The Debye relaxation time constant of bulk water is $\tau_{\mathrm{D}}=8.27$ ps. $\Delta G^{*}=$ $1.5 k_{\mathrm{B}} T$.
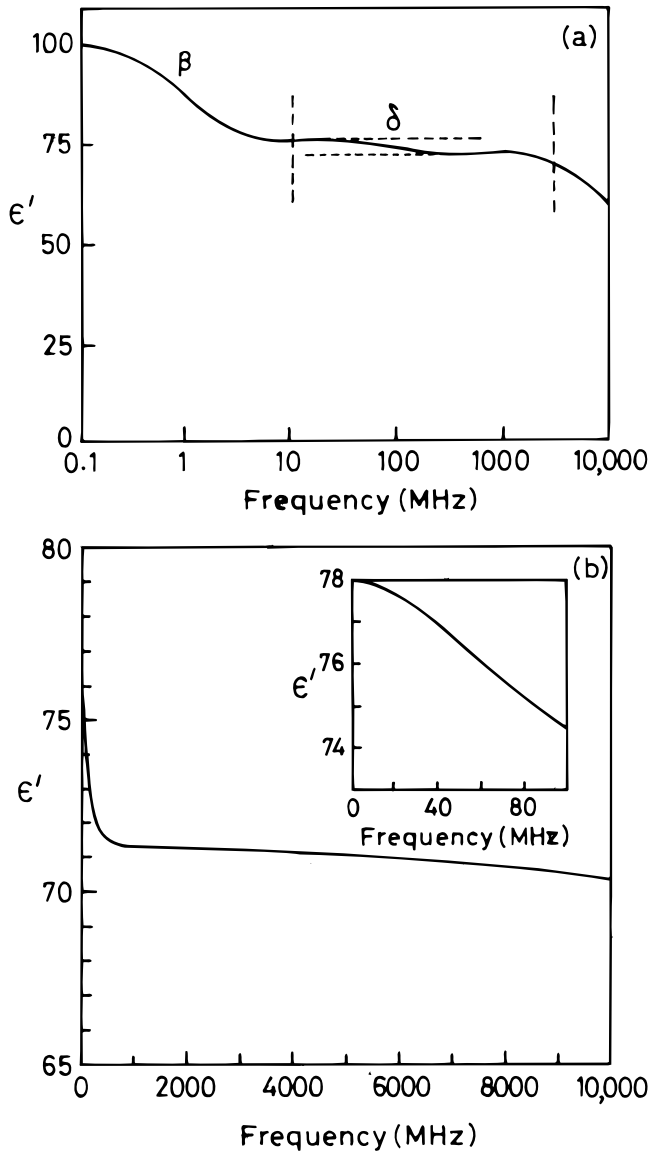

Figure 6. Plot of the real part of the frequency dependent dielectric function of biological water $\left(\epsilon^{\mathrm{B}}(\omega)\right)$ against frequency. Part a is taken from ref $8 \mathrm{~h}$. The area indicated by dashed lines represents the relaxation regime that is of interest in the present study. Part $b$ shows the real part of the frequency dependent dielectric function calculated from the present theory (see section IV). The time constants $\tau_{1}$ and the $\tau_{3}$ are $10.3 \mathrm{~ns}$ and $40 \mathrm{ps}$, respectively. ${ }^{8 \mathrm{~h}}$ The rest of the parameters are the same as in Figure 5.

attached to the protein at different polar groups. ${ }^{21}$ We can estimate the residence times of hydration water for different hydrogen bond strengths. The values calculated from the rate constants presented in the Table 2 are within the nano- to 


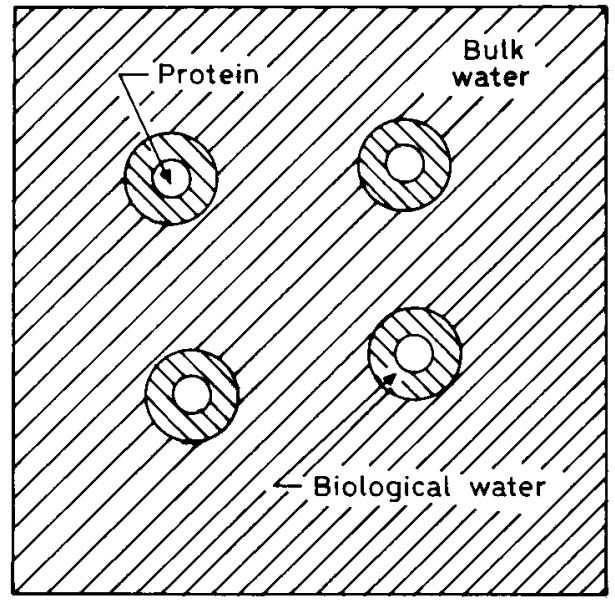

Figure 7. Schematic representation of a protein solution for which the moment - moment correlation function is given by eq 37 . For details see section VII of the text.

subnanosecond regime. It has been observed that the residence time of water of hydration increases with increasing hydrogen bond strength of the amino group.

\section{Relationship to the Conventional Dielectric Experiments on Protein Solutions}

The above theoretical discussion and the numerical calculations are applicable only to the biological water surrounding the biomolecule. In reality, however, these biomolecules exist in solution. Therefore, one cannot isolate the contribution of the biological water to the observed dielectric relaxation associated with the bulk water and the biomolecule. In fact, this has been a stumbling block in our understanding of biological water, since the bulk water often masks the dynamics of the biological water. Thus, one needs a careful fitting of the observed data, accounting for the different contributions. However, in order to have a successful fitting scheme, one needs to have a reliable theory for the relaxation of the total moment time correlation function. We now discuss how the theory developed here can be used to construct such a general theory, which can be applied to the understanding of the dielectric relaxation of real protein solutions.

First, note that any scheme of calculation of the frequency dependent dielectric functions for a solution of a biomolecule such as protein must include contributions from three sources: the protein rotation, the relaxation within the biological water (surrounding the protein and present within the hydration shell), and, of course, bulk water (see Figure 7). If one assumes that these contributions are uncorrelated with one other, then we can write the total moment-moment time correlation function as follows:

$$
\begin{array}{r}
C_{\mathrm{MM}}(t)=N^{2}\left[n_{\text {bulk }} \mu_{\mathrm{W}}{ }^{2} \mathrm{e}^{-t / \tau_{\mathrm{D}}}+\mu_{\mathrm{W}}{ }^{2}\left(w_{1} \mathrm{e}^{-t / \tau_{1}}+w_{2} \mathrm{e}^{-t / \tau_{2}}+\right.\right. \\
\left.\left.w_{3} \mathrm{e}^{-t / \tau_{3}}\left(\mathrm{e}^{-t / \tau_{\mathrm{B}}}\right)\right)+n_{\mathrm{B}}{ }^{2} \mu_{\mathrm{B}}{ }^{2} \mathrm{e}^{-t / \tau_{\mathrm{B}}}\right]
\end{array}
$$

where $N$ is the total number of the dipolar molecules in the system, i.e., the sum over protein molecules, biological water, and also bulk water molecules present in the system. $n_{\text {bulk }}=$ $N_{\text {bulk }} / N$ and $n_{\mathrm{B}}=N_{\mathrm{B}} / N$ are the respective number fractions of bulk water and the biomolecule (protein) in the system. $w_{1}=$ $\left(N_{\mathrm{f}} / N\right)^{2} C_{1}, w_{2}=\left(N_{\mathrm{f}} / N\right)^{2} C_{2}$, and $w_{3}=\left(N_{\mathrm{b}} / N\right)^{2} . \mu_{\mathrm{W}}$ and $\mu_{\mathrm{B}}$ are the dipole moments of bulk water and the biomolecule, respectively. Here, the dipole moment of biological water has been assumed to be the same as that of bulk water. In this case, the calculation of the frequency dependent dielectric function can be carried out using spherical geometry. ${ }^{33}$

However, the study of the dielectric behavior of the protein solution from $C_{\mathrm{MM}}(t)$ is difficult owing to uncertainties in the number of different species involved in the calculation. Nevertheless, one can at least qualitatively predict the features of the dielectric spectrum. The relaxation behavior of the second term in $C_{\mathrm{MM}}(t)$ (which represents the contribution of biological water) is expected to be affected significantly by the contribution of bulk water (in the high-frequency regime), as well as the protein motion (in the low-frequency regime). The bulk water contribution is expected to be large ( $N_{\text {bulk }}$ is very large) and dominant. The contribution to the relaxation from the orientational motion of the protein should also be significant, owing to the large dipole moment of the protein. ${ }^{34}$ Thus, if we calculate the frequency dependent dielectric function from $C_{\mathrm{MM}^{-}}$ $(t)$, it is expected that the contribution from biological water should lie between the fast motions of the bulk water and the slow motions of the protein. This is exactly the so-called " $\delta$ dispersion" observed in conventional dielectric measurements. ${ }^{8 \mathrm{k}}$ However, note that dominance of the relaxation spectrum by the bulk water makes experimental studies difficult.

\section{Conclusion}

Let us first summarize the main results of the present study. We have shown that the typical bimodal behavior of the dielectric relaxation of water associated with the biomolecules can be explained in terms of dynamic exchange between free and bound water species and the strength of the hydrogen bonds. The dynamic model shows that only free water contributes to the relaxation process, and the observed slow relaxation depends substantially on the strength of the hydrogen bonds.

The theory presented here has two distinct parts. First, the time dependence of the total dipole moment time autocorrelation function has been determined by using a kinetic model of interconversion between free and bound water at the surface of the biomolecule. The parameters used to evaluate the kinetics of this model are in agreement with previously obtained results. For example, the enhanced stability of the bound water relative to free water is reproduced correctly and so is the residence time of bound water. The most important aspect of our results is the bimodal nature of the moment-moment correlation function; the two time constants are separated by orders of magnitude, in good agreement with the known results. In the second part we used an extension of the continuum model to study the frequency dependent dielectric function of the biological water. The calculated Cole-Cole plot shows nicely the bimodality of dielectric relaxation. We have also formulated a theory of the dielectric relaxation of protein solutions. Although the detailed quantitative analysis of this model is difficult owing to the uncertainties involved in the experimental parameters used as input to the theory, we can understand the general qualitative features of the dielectric relaxation of protein solutions.

As already mentioned, this appears to be the first theoretical study of the dielectric relaxation of biological water. This study reveals that the dielectric relaxation in the complex biomolecular system depends on many parameters, such as the strength of the hydrogen bonds, the protein orientational relaxation time, the dipole moment of the protein, the values of the static and infinite frequency dielectric constant of biological water, etc. Although a complete theory of dielectric relaxation of protein solutions is far from complete, the success of the present approach is an encouraging step toward a successful theory of dielectric relaxation. 
Acknowledgment. Financial support from Department of Science and Technology, New Delhi is thankfully acknowledged. We thank an anonymous referee for constructive criticisms and corrections.

\section{References and Notes}

(1) Pethig, R. Аnnu. Rev. Phys. Chem. 1992, 43, 177.

(2) Rupley, J. A.; Careri, G. Adv. Protein Chem. 1991, 41, 37.

(3) Kuntz, I. D., Jr.; Kauzmann, W. Adv. Protein Chem. 1974, 28, 239.

(4) (a) Saenger, W. Annu. Rev. Biophys. Biophys. Chem. 1987, 16, 93. (b) Teeter, M. M. Annu. Rev. Biophys. Biophys. Chem. 1991, $20,577$.

(5) (a) Pethig, R. In Protein Solvent Interactions; Gregory, R. B., Ed.; Marcel Dekker Inc.: New York, 1995; Chapter 4, p 265 ff. (b) Eden, J.; Gascoyne, P. R. C.; Pethig, R. J. Chem. Soc., Faraday Trans. 1 1980, 76. 426. (c) Bone, S.; Eden, J.; Gascoyne, P. R. C.; Pethig, R. J. Chem. Soc., Faraday Trans. 1 1981, 77, 1729. (d) Bone, S.; Gascoyne, P. R. C.; Pethig, R. J. Chem. Soc., Faraday Trans. 1 1980, 76, 426. (e) Pethig, R. Dielectric and Electronic Properties of Biological Materials; Wiley: Chichester, 1979.

(6) (a) Oncley, J. L. J. Am. Chem. Soc. 1938, 60, 1115. (b) Oncley, J. L. In Proteins, Amino Acids and Peptides; Cohn, E. J., Edsall, J. T., Eds.; Reinhold: New York, 1943; Chapter 22.

(7) (a) Buchanan, T. J.; Haggis, G. H.; Hasted, J. B.; Robinson, B. G. Proc. R. Soc. London A 1952, 213, 379. (b) Haggis, G. H.; Buchanan, T. J.; Hasted, J. B. Nature (London) 1951, 167, 607.

(8) (a) Grant, E. H. Phys. Med. Biol. 1957, 2, 17. (b) Grant, E. H. Nature (London) 1962, 196, 1194. (c) Grant, E. H. Ann. N. Y. Acad. Sci. 1965, 125, 418. (d) Grant, E. H. J. Mol. Biol. 1966, 19, 133. (e) Grant, E. H.; Keefe, S. E. Rev. Sci. Instrum. 1968, 39, 1800. (f) Grant, E. H.; Keefe, S. E.; Takashima, S. J. Phys. Chem. 1968, 73, 4373. (g) Grant, E. H.; South G. P.; Takashima, S.; Ichimura, H. Biochem J. 1971, 122, 691. (h) Grant, E. H.; Mitton, B. G. R.; South, G. P.; Sheppard, R. J. Biochem. J. 1974, 139, 375. (i) Grant, E. H.; Sheppard, R. J.; South, G. P. In Dielectric Behavior of Biological Molecules in Solutions; Clarendon: Oxford, 1978. (j) Essex, C. G.; Symonds, M. S.; Sheppard, R. J.; Grant, E. H.; Lamote, R.; Sosseneu, M. Y.; Peeters, H. Phys. Med. Biol. 1977, 22, 1160. (k) Grant, E. H.; McLean, V. E. R.; Nightingale, N. R. V.; Sheppard, R. J.; Chapman, M. J. Bioelectromagnetics 1986, 7, 151.

(9) (a) Schwan, S. P. Ann. N. Y. Acad. Sci. 1965, 125, 344. (b) Pennock, B. E.; Schwan, S. P. J. Phys. Chem. 1969, 73, 2600.

(10) (a) Takashima, S. J. Mol. Biol. 1963, 7, 455. (b) Takashima, S. J. Phys. Chem. 1966, 70, 1372. (c) Takashima, S. Adv. Chem. Ser. 1967, 63, 232. (d) Takashima, S. In Physical Principles and Techniques of Protein Chemistry; Leach, S. J., Ed.; Academic Press: New York, 1969; Part A, p 291 ff. (e) Takashima, S.; Gabriel, C.; Sheppard, R. J.; Grant, E. H. Biophys. J. 1984, 46, 29. (f) Takashima, S.; Casaleggio, A.; Giuliano, F.; Morando, M.; Arrigo, P.; Ridella, S. Biophys. J. 1986, 49, 1003. (g) Takashima, S. In
Recent Progress in the Dielectric Research on Polynucleotides, Protides of the Biological Fluids; Peeters, H., Ed.; p 367.

(11) Harvey, S. C.; Hoekstra, P. J. Phys. Chem. 1972, 76, 2987.

(12) Brey, W. S., Evans, T. E.; Hitzrot, L. H. J. Colloid Interface Sci. 1968, 26, 306

(13) Singh, G. P.; Parade, F.; Huklinger, S.; Dransfeld, K. Phys. Rev. Lett. 1981, 47, 685 .

(14) (a) Mashimo, S.; Kuwabara, S.; Yagihara, S.; Higasi, K. J. Phys. Chem. 1987, 91, 6337. (b) Kuwabara, S.; Umehara, T.; Mashimo, S.; Yagihara, S. J. Phys. Chem. 1988, 92, 4839. (c) Mashimo, S.; Umehara, T.; Kuwabara, S.; Yagihara, S. J. Phys. Chem. 1989, 93, 4963. (d) Fukuzaki, M.; Miura, N.; Shinyashiki, N.; Kurita, D.; Siyoa, S.; Munetaka, H.; Mashimo, S. J. Phys. Chem. 1995, 99, 431.

(15) (a) Gestblom, B. J. Phys. Chem. 1991, 95, 6064. (b) Gestblom, B.; Noreland, E. J. Phys. Chem. 1984, 88, 664.

(16) Fullerton, G. D.; Ord, V. A.; Cameron, I. L. Biochim. Biophys. Acta 1986, 230.

(17) Lioutas, T. S.; Baianu, I. C.; Steinberg, M. P. Arch. Biochem. Biophys. 1986, 247, 68 .

(18) (a) Peemoeller, H.; Shenoy, R. K.; Pintar, M. M. J. Magn. Reson. 1981, 45, 193. (b) Peemoeller, H.; Kydon, D. W.; Sharp, A. R.; Schreiner, L. J. Can. J. Phys. 1984, 62, 1002. (c) Peemoeller, H.; Yeomans, F. G.; Kydon, D. W.; Sharp, A. R. Biophys. J. 1986, 49, 943.

(19) Usha, M. G.; Wittebort, R. J. J. Mol. Biol. 1989, 208, 669.

(20) Hagler, A. T.; Moult, J. Nature 1978, 272, 222.

(21) Otting, G.; Liepinsh, E.; Wuthrich, K. Science 1991, 254, 974.

(22) (a) Bryant, R. G. Annu. Rev. Phys. Chem. 1978, 29, 167. (b) Bryant, R. G. In The Chemical Physics of Solvation; Dogonadze, R. R., Kalman, E., Kornyshev, A. A., Ulstrup, J., Eds.; Elsevier: Amsterdam, 1988; Chapter 14, p $683 \mathrm{ff}$.

(23) Polnaszek, C. F.; Bryant, R. G. J. Am. Chem. Soc. 1984, 106, 428.

(24) Hendrickx, H.; Verbruggen, R.; Rosseneu-Mottreff, M. Y.; Blaton, V.; Peeters, H. Biochem. J. 1968, 110, 419.

(25) Oakes, J. J. Chem. Soc., Faraday Trans. 1 1972, 72, 216.

(26) Halle, B.; Andersson, T.; Forsen, S.; Lindman, B. J. Phys. Chem. 1981, 103, 500 .

(27) (a) Koenig, S. H.; Hallenga, K.; Shporer, M. Proc. Natl. Acad. Sci. U.S.A. 1975, 72, 2667. (b) Koenig, S. H.; Schillinger, W. E. J. Biol. Chem. 1969, 244, 3283. (c) Koenig, S. H. ACS Symp. Ser. 1980, 127, 157.

(28) Grosch, L.; Noack, F. Biochim. Biophys. Acta. 1976, 453, 218.

(29) Titulaer, U. M.; Deutch, J. M. J. Chem. Phys. 1974, 60, 1502.

(30) Frohlich, H. Theory of Dielectrics; Clarendon: Oxford, 1958.

(31) Laidler, K. J. Chemical Kinetics; Harper and Row: New York, 1987.

(32) Jeffrey, G. A.; Saenger, W. Hydrogen Bonding in Biological Systems; Springer Verlag: New York, 1991; Chapter 2, p 28 ff.

(33) Zwanzig, R. W. J. Chem. Phys. 1963, 38, 2766.

(34) Antosiewicz, J. Biophys. J. 1995, 69, 1344. 